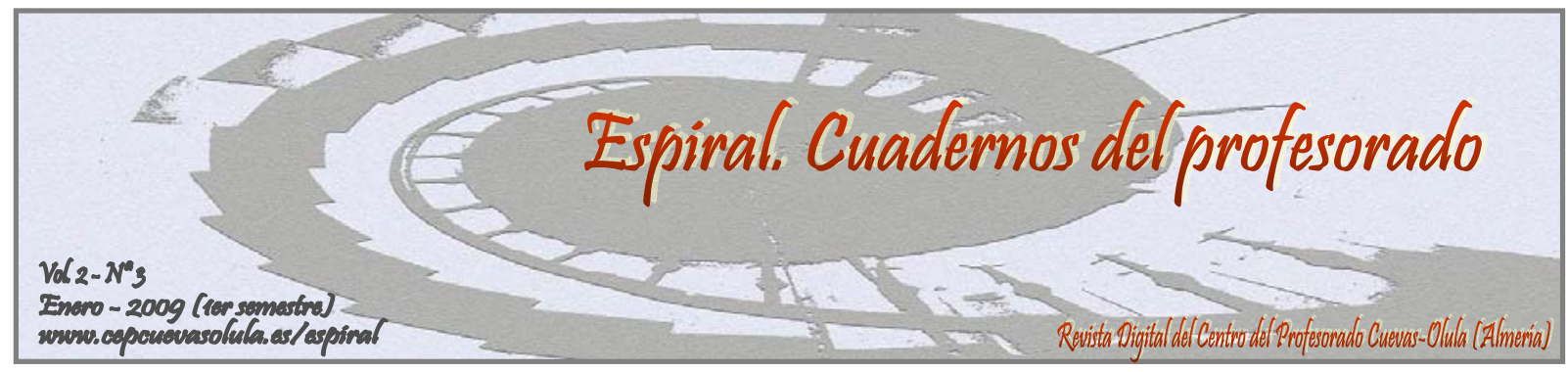

\title{
LAS PIRUETAS Y SU APLICACIÓN EN DIFERENTES DISCIPLINAS DEPORTIVAS
}

\section{THE PIROUETTES AND THEIR APPLICATION IN DIFFERENT SPORTING DISCIPLINES}

\section{Elena Conesa Ros}

\section{Facultad de Ciencias del Deporte. Universidad de Murcia (España)}

Conesa Ros, E. (2009). Las piruetas y su aplicación en diferentes disciplinas deportivas [en línea]. Espiral. Cuadernos del Profesorado, 2(3), 74-81. Disponible en: http://www.cepcuevasolula.es/espiral.

Enviar correspondencia a: econesaros@um.es

RESUMEN: El propósito de este estudio es realizar una revisión bibliográfica del término pirueta y sus diferentes acepciones en algunas disciplinas artístico-deportivas. Profundizaremos en la terminología, definición y clasificación del elemento pirueta, examinando las diferencias que existen en su ejecución en las diferentes disciplinas deportivas analizadas (Gimnasia Rítmica, Gimnasia Artística, Patinaje Artístico y Natación Sincronizada). Dichas disciplinas están relacionadas con el mundo del arte, el baile y la música; es por esto que en ellas se realiza uno de los movimientos más bellos y estéticos que recibe el nombre de pirueta. Igualmente en este trabajo se proponen ejercicios para la mejora de la ejecución técnica de la pirueta.

Palabras clave: pirueta, giros, vueltas, tours, equilibrio.

ABSTRACT: The purpose of this study is to conduct a literature review of the term pirouette and its different meanings in some artistic and sporting disciplines. Delve into the terminology, definition and classification of the element pirouette, considering the differences in their performance in various sports analyzed (Rhythmic Gymnastics, Artistic Gymnastics, figure skating and synchronized swimming). These disciplines are related to the world of art, dance and music, which is why they are one of the most beautiful and aesthetic movements that called the pirouette. Also in this study suggests exercises to improve the technical execution of the pirouette.

Key words: pirouette, twists, turns, tours, balance. 


\section{1.- ORIGEN DE LA PIRUETA.}

El origen de la palabra pirueta proviene del francés piruette, la cual viene a significar la tapa que hace girar, mientras que en cambio en el Ballet Clásico viene a significar dar vueltas sobre una pierna. Es un movimiento que los bailarines o deportistas utilizan para sorprender a su audiencia o jueces, girando más de una vez sobre el eje longitudinal. Se puede realizar en punta o media punta (encima de la punta clásica que utiliza la bailarina o apoyando solo los metatarsos del pie). Si se realiza en media punta el bailarín debe usar todos los dedos del pie de soporte, presionando fuertemente contra la tierra y aumentando el área de ayuda. Las piruetas requieren un equilibrio perfecto y dependen íntegramente de la preparación previa.

Geneive, Guillot y Germaine (1974) llaman piruettes (piruetas) a los giros en los cuales el cuerpo, descansando sobre un solo pie, hace uno o varios giros sobre sí mismo, sin que el pie de apoyo tenga movimiento propio.

Los "tours de promenade" en los cuales el pie de apoyo produce el movimiento mediante cortos desplazamientos, no pueden, pues, clasificarse como pirueta, ya que en estas últimas el impulso se realiza de una sola vez al principio mediante todo el conjunto del cuerpo.

\section{2.- LAS PIRUETAS EN LA DANZA.}

Las vueltas son un elemento universal de la Danza, aunque muchos pueblos no conozcan más que las vueltas sobre los dos pies, debido a que las que se ejecutan sobre un pie exigen una combinación muy consciente de la posición de los brazos, del cuerpo y de la cabeza.

Una de las habilidades específicas más llamativa de la danza a la hora de ser observada por el espectador es la pirueta, junto con los saltos. Algunos bailarines, haciendo uso de una buena técnica, llegan a realizar más de una pirueta seguida asemejándose a una peonza, dejando al espectador ensimismado. Para su ejecución, además de la estructura corporal (proporción hombro-caderas) influyen una serie de factores como la fuerza de rozamiento del pie con el suelo, los segmentos corporales (brazos, pierna libre y cabeza), la colocación del cuerpo, etc. que hacen aún más difícil su correcta ejecución. Señalar también que cuando se aprende a girar correctamente, se obtiene una sensación tan motivante y placentera que no se querría dejar de hacer nunca.
Algunos bailarines parecen progresar en cualquier otro aspecto de su danza excepto en sus giros, debido probablemente a que han establecido un patrón de movimiento erróneo a base de practicarlo durante años. En muchos casos, es más fácil ayudar a un principiante que a un estudiante avanzado, cuyos hábitos están arraigados más firmemente, siendo estos más difíciles de cambiar.

Para adentrarnos en el complicado mundo de las piruetas, hay que mencionar que éstas pertenecen al grupo de las vueltas, las cuales según Guillot y Prudhommeau (1974) implican una rotación alrededor del cuerpo. Estas pueden ser ejecutadas sobre los dos pies, un pie o saltando. Los dos primeros tipos se caracterizan por un contacto constante de los pies con el suelo y se clasificarán en los "tours á terre" (vueltas con apoyo en el suelo). Estas vueltas son dinámicas por naturaleza y en la mayoría de los casos, la posición del cuerpo no varía durante la ejecución de la rotación.

En cuanto a la terminología, como podemos observar, la mayoría de autores utilizan indistintamente el término giro o vuelta para referirse al mismo concepto. Para Elena Sierra (1992), dentro de los giros ella hace la siguiente distinción:

$\square$ Vueltas: giros que utilizan como base de apoyo los dos pies.

$\square$ Giros (o pivotes): giros en los que la base de apoyo es un solo pie.

Las grandes piruetas son comúnmente denominadas tours, ejemplo de ello es una pirueta en attitude, tour en attitude ó aquellas que son realizadas en series, como el tour piqué. Para continuar con la profundización de estos términos, debemos introducirnos en aquellas vueltas cuya rotación es efectuada sobre un solo pié. Entre ellas encontramos aquellos giros que se llevan a cabo con todo el pié apoyado, en media punta 0 en punta completa (Guillot y Prudhommeau, 1974). Mientras que normalmente, los giros lentos pueden ejecutarse únicamente con el pié apoyado, los rápidos en cambio se llevan a cabo en punta por las mujeres $\mathrm{y}$ en media punta por los varones.

Centrándonos en los giros, éstos pueden iniciarse desde distintas posiciones tomadas del Ballet Clásico, siendo las más comunes $2^{\mathrm{a}}, 4^{\mathrm{a}} \mathrm{o}$ $5^{\text {a }}$. Estos pueden realizarse en los dos sentidos de rotación (En Dedans y En dehors) variando la posición de la pierna libre. En las danzas de 
carácter se pueden encontrar vueltas a pie plano o sobre el talón.

\section{3.- LAS PIRUETAS EN LA GIMNASIA RÍTMICA.}

Mientras que en la danza, se diferencia entre giro y pirueta, en la Gimnasia Rítmica le suelen llamar a todo indistintamente giros. Según la Diputación de Orense (1991) los giros no son elementos rígidos, predeterminados, que no aceptan variación alguna, sino que son un grupo de elementos abiertos a la originalidad y creatividad de la entrenadora. Uno de los aspectos que diferencian a la pirueta de la Gimnasia Rítmica de la que se realiza en Ballet, es que la pierna libre puede estar rígida o flexionada, y que las posiciones del tronco y de la cabeza pueden variar, no importando su posición, siempre que ésta posea un aspecto agradable. Estos mismos autores clasifican los giros en semigiros $\left(180^{\circ}\right)$, giros completos $\left(360^{\circ}\right)$ y dobles giros $\left(720^{\circ}\right)$.

Su técnica es similar a la que se realiza en Ballet, con algunas excepciones:

- La pierna sobre la cual se realiza el giro en algunos casos se dobla

- Con la colocación del cuerpo hay que tener cuidado, pues por el abuso de la flexibilidad las gimnastas tienden a curvarse

- En gimnasia es difícil determinar la posición de los brazos, debido a que hay que llevarlos en función del aparato

- El movimiento de la cabeza es similar con la salvedad de las piruetas en attitude las cuales deben seguir la trayectoria del giro

Es interesante mencionar que en Gimnasia Rítmica existe una serie de faltas en la ejecución de los giros que tienen sus correspondientes penalizaciones:

- Elevación insuficiente en los giros sobre la punta $(0,10)$

- Apoyo pasajero del talón durante un giro sobre la punta $(0,20)$

- Giro incompleto $(0,10)$

- Pérdida de equilibrio al final de un giro $(0,20)$

El trabajo práctico que propone la Diputación de Orense (1991) es el siguiente:

- Toma de conciencia de la postura corporal correcta para el equilibrio y el giro

- Énfasis en contraer-relajar los adecuados grupos musculares
- Trabajo de elevación máxima de la media punta combinando plie-releve en las cinco posiciones clásicas $\left(1^{\mathrm{a}}, 2^{\mathrm{a}}, 3^{\mathrm{a}}, 4^{\mathrm{a}}\right.$ y $\left.5^{\mathrm{a}}\right)$

- Trabajo en la barra de equilibración en las distintas posiciones en las que se ejecutaran los giros

- Trabajo de giros en la barra

- Trabajo de giros en el centro

El trabajo se llevará a cabo por parejas, sobre todo en aquellas posiciones que precisen una corrección de la colocación corporal. Mendizábal y Mendizábal (1985) señalan que en Gimnasia Rítmica, durante un giro se puede variar la postura de la pierna lo que aumenta su dificultad. Además, aunque ya no se le denominarían piruetas, en Gimnasia Rítmica también se realizan giros sobre glúteos, espalda, etc.

Heinss (1991) presenta una serie de ejercicios para conseguir una correcta ejecución de los giros (donde incluiríamos a la pirueta) que no aparecen anteriormente y en los que presta especial atención a la orientación correcta en el espacio:

- Interrumpir el caminar lento y rápido mediante vueltas rápidas en el mismo lugar y plantear tareas de reacción. Hacer ejecutar, a una señal, una o varias vueltas rápidas; a continuación sigue el movimiento adelante

- Combinar en forma cíclica las vueltas caminadas con un correr lento y rápido. Duplicar también el ritmo de las vueltas, hacer ejecutar dos vueltas rápidas en el mismo tiempo, luego seguir con seguridad rectilíneamente

- Caminar adelante y atrás en posición tensa en combinación con media vuelta rápida: Compás de 4 tiempos $=8$ pasos adelante, 8 pasos atrás, al realizar el $7^{\circ}$ y el $8^{\circ}$ paso dar media vuelta

- ¿Quién puede seguir caminando derecho después de haber realizado varias vueltas rápidas en un mismo lugar?

- Realizar de 3 a 5 vueltas rápidas en un mismo lugar en posición semiflexionada, luego seguir caminando con seguridad sobre una línea trazada con tiza hasta un punto determinado. ¿Quién es la que menos se aparta de la línea? Con estos ejercicios se entrena el sentido del equilibrio y la capacidad de orientación

Según Canalda (1998) resulta fundamental trabajar los giros mediante la utilización de la barra de ballet o espalderas, por ello indica que se 
puede recurrir a la lectura de obras específicas de ballet clásico para enseñarlos de manera correcta. En su progresión hacia los giros primero comienza con los brazos en cruz y luego en la cintura.

Para Brikina (1978) los giros en Gimnasia Rítmica son ejercicios peculiares de equilibrio que se realizan en media punta de un pie con diferentes posiciones de la pierna libre, brazos y tronco. Bodo-Schmid (1985) la llama pirueta y no giro y la define como un movimiento rápido de trompo sobre un pie. Martínez (1992) denomina vueltas al paso intermedio entre el equilibrio y el giro los cuales son realizados sobre la punta de los pies.

Esta misma autora añade a la serie de ejercicios para la progresión de la pirueta, los siguientes:

- Realizar un giro en la barra de $360^{\circ}$ en 4 partes, o sea, girando $45^{\circ}$ cada vez

- Pasar a realizar el giro con ayuda de la barra en dos partes, o sea, $180^{\circ}$ cada vez

- Realizar el giro de $360^{\circ}$ en una sola vez

- Repetir la progresión fuera de la barra

Según Fernández (1989) el término pirueta está cayendo en desuso por el empleo de la palabra giro. Asimismo comenta que el peso el cuerpo en la cuarta posición antes de ejecutar la pirueta debe estar sobre ambas piernas, ligeramente adelantado. Por último realiza la siguiente progresión orientativa (tabla 1).

O’Farrill y Santos (1982) denominan vueltas a los ejercicios que se realizan alrededor de un eje imaginario, situado fuera del cuerpo del ejecutante. Se trata de elementos de fácil ejecución, muy relacionados con el grupo de pasos y carreras, pues se ejecutan realizando un recorrido que describe una circunferencia, ya sea caminando o corriendo. Las vueltas tienen como objetivo el desarrollo de la orientación espacial, la cual radica en el aparato vestibular, situado en el oído interno. Por este motivo son ellas las indicadas para iniciar a las principiantes en el estudio de este grupo. Las vueltas son, por tanto, muy útiles para crear las condiciones iniciales que preparan a las alumnas para la realización de los giros. Su requerimiento de ejecución es tan simple que permite su empleo en las primeras clases sin distinción de edad.

Aunque existen giros cuyo nivel de ejecución no presenta grandes complejidades, lo cierto es que estos están estrechamente relacionados con el equilibrio y por supuesto, con la orientación espacial. En su ejecución suelen intervenir casi todos los elementos corporales, los cuales realizan funciones específicas para el

Tabla 1.- Progresión orientativa.

\section{7 a 8 AÑOS}

- Elaborar la elasticidad en barra “fijando el eje del aplomo en la espina dorsal”, según Vaganova

- Preparación de pirueta en passé cerrado desde sexta posición en dehors hasta $90^{\circ}$

\section{8 a 9 AÑOS}

- Elaborar la elasticidad en barra "fijando el eje del aplomo en la espina dorsal”, según Vaganova

- Preparación de pirueta en passé cerrado desde sexta posición en dehors hasta $90^{\circ}$

- Pirueta simple en passé cerrado desde sexta posición

- Preparación de piruetas sin releve desde tercera posición

- Preparación de piques en la barra

\section{9 a 10 AÑOS}

- Elaborar la elasticidad en barra "fijando el eje del aplomo en la espina dorsal”, según Vaganova

- Preparación de pirueta en passé cerrado desde sexta posición en dehors hasta $90^{\circ}$

- Pirueta simple en passé cerrado desde sexta posición

- Preparación de piruetas sin releve desde tercera posición

- Preparación de piques en la barra

- Pirueta en dehors con releve desde tercera o quinta posición

- Serie de piques sin girar

- Preparación de la pirueta en dedans en barra

- Serie combinada de los ejercicios anteriormente descritos

- Preparación pirueta en dehors desde cuarta posición

- Pirueta en dehors, desde cuarta posición

- Preparación de pirueta en dedans desde cuarta posición 
logro del cumplimiento del elemento.

El impulso es un factor esencial en el giro. Mientras que la gimnasta no aprenda a tomar impulso y a aprovecharlo en todo su dimensión, no logrará la ejecución correcta de este elemento. También debe insistirse en que el impulso no debe ser exagerado, ni basarse en posiciones estáticas previas, como sucede en el ballet, donde las piruetees son precedidas de una preparación. El impulso brindado por las piernas puede realizarse mediante un paso previo de la pierna de apoyo o por resorte de esta misma pierna; la pierna libre también puede cooperar con el impulso según la posición que adopte o el movimiento que realice durante el giro.

Estos autores clasifican los giros por grados, lo cual permite determinar su recorrido con exactitud cuando se señalan en una selección o en una lista de elementos de dificultad. Se llaman giros simples a todos aquellos que son menores o iguales a $360^{\circ}$ y complejos a los que son mayores de $360^{\circ}$. Esta clasificación de los giros según los grados, cuenta entre los giros simples mas frecuentes, los de $90^{\circ}, 135^{\circ}, 180^{\circ}, 225^{\circ}, 270^{\circ}$ y $360^{\circ}$, mientras que en los giros complejos se utilizan los $540^{\circ}, 720^{\circ}$ y excepcionalmente los de $1080^{\circ}$.

Los giros sobre un pie se dividen en simétricos y asimétricos. Se denomina giro simétrico a aquel que se efectúa hacia el mismo lado de la pierna de apoyo, por ejemplo, cuando se gira sobre el pie derecho hacia la derecha. El giro asimétrico, en cambio, es el que se realiza al lado contrario de al pierna de apoyo. Este último es de más fácil ejecución, por lo que no se necesita de una asimilación previa, como pasaría con el simétrico.

Al aparecer el nuevo concepto de vuelta, O’Farrill y Santos (1982) proponen los siguientes ejercicios para la mejora de las piruetas:

- Vuelta en ocho pasos al frente: desde la sexta o tercera posición, salir con el pie simétrico hacia donde se indica la realización de la vuelta, y describir una circunferencia alrededor de un eje imaginario, situado a un costado de la ejecutante. Dar siempre pasos de marcha gimnástica o de carrera, mirando por encima del hombro, y en el octavo paso unir los pies en el lugar inicial. Los brazos pueden comenzar y terminar laterales, pero durante la ejecución cambian de posición.

Errores comunes:

- No realizar el punteo en los pasos

- No mirar sobre el hombro
- No llegar al lugar en siete pasos

- No acabar los brazos en posición correcta

- Vuelta en ocho pasos atrás: es similar a la anterior, pero se pierde más fácilmente la orientación en el espacio. Los pasos se dan hacia atrás y los brazos se mantienen en posición preparatoria.

\section{Errores comunes:}

- No regresar al lugar inicial

- No mirar sobre el hombro

- Vuelta en forma de ocho: se realiza en 16 tiempos; consiste en combinar una vuelta a la derecha con una a la izquierda, o viceversa, cambiando la posición de los brazos y de la cabeza sin detenerse en el centro. Los pies se unen en el lugar inicial en el tiempo 16. Se hace caminando o corriendo.

Errores comunes:

- No ajustarse a los pasos requeridos

- No hacer el cambio de brazos en el momento adecuado (tiempo 8 ó 9)

- Descuidar la posición de los brazos

- Giro con pequeños pasos en el lugar: puede comenzarse en distintas posiciones, emplearse diversos movimientos de brazos, finalizar en forma variada, etc., pero el elemento consiste en elevar los talones y mediante pasos muy pequeños, despegando poco el metatarso del piso y las piernas extendidas, ir girando hasta la cantidad de grados deseada.

Errores comunes:

- Levantar mucho los pies del piso

- Flexionar las piernas

- No girar en la gradación que se había orientado

- No cuidar la posición o el movimiento de los brazos

- Giro cruzado: desde la sexta o tercera posición un pie cruza sobre el otro, se elevan los talones al máximo y se efectúa el giro cuidando que al final los pies no queden cruzados sino uno al lado del otro.

Gula (1990) denomina a las piruetas en dehors piruetas hacia dentro y a las en dedans piruetas hacia fuera. Aparo y Cermeli (1995) dividen las piruetas en normal e inversa, términos similares al en dehors y en dedans.

Pica (1988) diferencia entre el termino passé en ballet y en gimnasia. En ballet esta palabra significa "pasado" y generalmente la rodilla de la pierna de soporte es pasada por la pierna de 
trabajo cuando la bailarina se esta moviendo de una posición a otra. Passé puede también referirse al paso de las piernas por el aire o al movimiento en la cual una pierna es elevada y pasada por delante o por detrás de la pierna de soporte. En gimnasia el termino passé es a menudo usado de manera incorrecta para definir el actual passé y el retiré. Estos términos no son intercambiables, sin embargo passé es un movimiento en el cual la rodilla es pasada, y retiré es una posición estática en la cual el pie de la pierna de trabajo está situado en la rodilla de la de soporte.

Bobo y Sierra (1988) entienden por giro la rotación del cuerpo alrededor de un eje longitudinal, teniendo como punto de apoyo alguna superficie corporal. Los giros en los que la base del giro es un solo pie los denominan pivotes.

Tanto los giros en dedans como los en dehors se emplean en gimnasia, con preferencia de uno u otro según la técnica individual de cada gimnasta; pero en general cuando se quiere conseguir rotación de muchos grados $720^{\circ}$ o más, se suelen utilizar los giros en dedans.

Es importante resaltar que la acción de los brazos debe ser suave evitando los movimientos bruscos o latigazos ya que estos muchas veces son la causa del desequilibrio en los giros. A menudo las gimnastas quieren buscar la velocidad de rotación en la fuerza de los brazos, ya que el resultado es un giro muy impreciso y difícil de controlar lo que produce una perturbación general en el equilibrio de la espalda que es esencial en las rotaciones.

\section{4.- LAS PIRUETAS EN LA GIMNASIA ARTÍSTICA.}

Brüggemann (1994) divide los elementos dinámicos de la gimnasia en cinco categorías:

- Categoría 1: TAKO- Despegar e irse desde una sólida o elástica superficie (óptima producción de la velocidad de despegue del cuerpo).

- Categoría 2: ROVE- Rotaciones en el plano vertical sobre un flexible y fijo eje horizontal de rotación (óptima producción y uso de la energía mecánica).

- Categoría 3: ROHO- Rotaciones en un plano horizontal sobre el eje vertical de rotación.
- Categoría 4: AIRO- Rotaciones en el aire (cambios óptimos en momentos de inercia).

- Categoría 5: LAND- Aterrizaje (máxima absorción de la energía mecánica).

Las piruetas, al ser rotaciones sobre el eje longitudinal, son también elementos de la categoría ROHO. En todos estos elementos, la fuerza gravitatoria actúa perpendicular al plano de movimiento. En este grupo, el peso del cuerpo nunca funciona como un generador del movimiento; la fuerza de los músculos permanentes debe actuar contra la gravedad sin ningún efecto en detrimento del movimiento. El movimiento de rotación, este es, el momento angular del eje vertical, debe ser producido por fuerzas horizontales y momentos sobre el eje vertical.

\section{5.- LAS PIRUTETAS EN EL PATINAJE ARTÍSTICO SOBRE RUEDAS.}

Para De Diego (1996) las piruetas son bucles girando en un punto, siendo éstas exteriores e interiores. Habitualmente las piruetas se realizan con vistas a facilitar las ejecuciones de enlace entre saltos, por ello se efectúan de la siguiente manera:

Para los diestros: giro lado izquierdo:

- Punta y talón

- Atrás interior, pie izquierdo

- Adelante exterior, pie izquierdo

- Atrás exterior, pie derecho

Para los zurdos: giro lado derecho:

- Punta y talón

- Atrás interior, pie derecho

- Adelante exterior, pie derecho

- Atrás exterior, pie izquierdo

Las piruetas constan de cierre y giro, que son los mismos que para los saltos. En todas ellas se cerrará de igual manera y su giro se realizará hacia el lado izquierdo para los diestros y derecho para los zurdos.

Existen varios tipos de piruetas: altas, bajas, agachadas, de ángel y torsionadas. Dentro de estas posiciones las hay muy variopintas, dependiendo de la habilidad y esfuerzo del patinador.

Para conseguir el giro en las piruetas es imprescindible, el levantar una rueda del patín que está en el suelo, además de una velocidad de entrada e impulso de la pierna libre en algunas 
piruetas. No se consideran como piruetas las que se realizan sobre el freno.

Las piruetas podemos clasificarlas según:

- La trayectoria: exterior adelante, exterior atrás, interior adelante e interior atrás

- La posición del cuerpo: ángel, bajas y altas

- El apoyo en dos ruedas: en las ruedas de atrás, en las interiores y en las exteriores

- La ejecución dentro de la pirueta: saltadas, combinadas, reversadas y desplazadas

\section{6.- LAS PIRUETAS EN LA NATACIÓN SINCROZADA.}

En natación sincronizada a los giros que se realizan bajo el agua no se les denominan piruetas sino Twists o Spins. Elkington y Chamberlain (1986) definen dichos conceptos de la siguiente manera: el Twists es una rotación con el cuerpo sostenido en la posición verticalinvertida, manteniendo la misma altura a través del movimiento. El nivel del agua está entre las rodillas y las caderas, y la cabeza debe estar en línea con el tronco. Así es posible enseñar: un medio Twists de $180^{\circ}$ y un Twists completo de $360^{\circ}$. La posición de los brazos es opcional. Un brazo necesita mantener la altura y la estabilidad y el otro añadir la rotación.

En cambio un Spin es una rotación descendiendo ejecutada en un movimiento uniforme. El cuerpo está en la posición de vertical-invertida. Éste debe ser absolutamente recto y firme con la cabeza en línea con el tronco. Todo Spins debería comenzar en la máxima altura vertical posible. Tanto para si son de $180^{\circ}$ como de $360^{\circ}$, el movimiento debe ser completado al tiempo en que los talones alcancen la superficie del agua.

Finalmente y a modo de conclusión, podemos afirmar que la mayoría de la bibliografía consultada sobre la pirueta, hace referencia sobre todo a las distintas definiciones existentes y no a los aspectos técnicos de su ejecución. Incluso el concepto de vuelta, giro o pirueta difiere dependiendo del autor consultado. Igualmente señalar que no hay unanimidad en cuanto a la progresión que hay que seguir para la realización de una correcta pirueta. Otra conclusión interesante es que se conocen los principios físicos que intervienen en la ejecución de la misma pero no su aplicabilidad. Por último, comentar que la ejecución técnica de las piruetas en otras disciplinas deportivas, a excepción de la
Gimnasia Rítmica se aleja muchísimo de las que se realizan en Ballet.

\section{7.- REFERENCIAS BIBLIOGRÁFICAS.}

Aparo, M. \& Cermeli, S. (1995). Il corpo libero nela ginnastica moderna. Roma: Societa' Stampa Sportiva.

Bobo, M. \& Sierra, E. (1998). A Ximnasia Rítmica Deportiva: Adestramento $e$ competición. Santiago de Compostela: Lea.

Bodo-Schmid, A. (1985). Gimnasia Rítmica Deportiva. Barcelona: Hispano Europea.

Brikina, A. T. (1978). Gimnasia. Zaragoza: Acribia.

Brüggemann, G-P. \& Sporthochschule, D. (1994). Biomechanics of Gymnastic Techniques. Sport Science Review, 3(2), 79-120.

Canalda, A. (1998). Gimnasia Rítmica Deportiva. Teoría y práctica. Barcelona: Paidotribo.

Diputación de Orense (1991). Jornadas Técnicas de Gimnasia Rítmica. Orense: Diputación de Orense. Federación Gallega de Gimnasia.

Elkington, H. \& Chamberlain, J. (1986). Synchronised Swimming. David \& Charles Inc. USA.

Fernández, A. (1989). Gimnasia Rítmica Deportiva: fundamentos. Madrid: Federación Española de Gimnasia.

Guillot, G. \& Prudhommeau, G. (1974). Gramática de la Danza. Buenos Aires: Hachette.

Gula, D. (1990). Dance Choreography for Competitive Gymnastics. Estados Unidos: Leisure Press.

Heinss, M. (1991). Gimnasia Rítmica Deportiva para niñas. Buenos Aires: Stadium.

Martínez Vidal, A. (1992). Cuadernos Técnicos de Deporte. La Gimnasia Rítmica: un planteamiento educativo motriz. Orense: Excma. Diputación Provincial de Orense. 
Mendizábal, S \& Mendizábal, I. (1985). O’Farrill, A. \& Santos, A (1982). Gimnasia Iniciación a la gimnasia rítmica (manos libres-cuerda-pelota). Madrid: Gymnos.

Rítmica Deportiva. Buenos Aires: Stadium.

Nanni, D. (1995). Dança Educaçao. Pica, R. (1988). Dance training for Princípios, Métodos e Técnicas. Río de Janeiro: Sprint.

Gymnastics. Estados Unidos: Leisure Press.

De Diego, N. (1996). Iniciación al patinaje artístico sobre ruedas. Madrid: Gymnos.

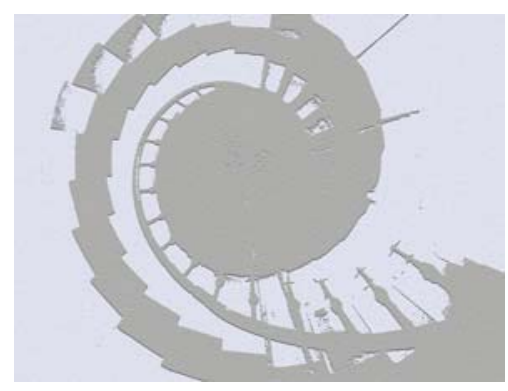

